\title{
Circulating secretory component in relation to early diagnosis and treatment of liver metastasis from colorectal carcinomas
}

\author{
D Kvale, J Norstein, G I Meling, O P Børmer, P Brandtzaeg, F Langmark,
} T O Rognum

\begin{abstract}
Aims: To evaluate serum secretory component in relation to early detection and clinical management of liver metastasis in patients with colorectal cancer.

Methods: Secretory component and carcinoembryonic antigen (CEA) were analysed in serial serum samples from 23 patients who had liver metastases as the only apparent recurrence, and in sera from 54 matched controls. Results of surgical treatment of recurrences were classified peroperatively as radical when no residual tumour was apparent and resection margins were free of disease.

Results: In total, $18(78 \%)$ patients had increased secretory component during the whole follow up period (median 16 months); 12 (52\%) had raised secretory component concentrations before clinical recurrence (median lead time 5.2 months). There was no difference before recurrence between circulating secretory component and CEA in sensitivity and lead times. Seventeen patients underwent surgery for hepatic metastasis; seven had radical hepatic resection of which only two (29\%) showed increased secretory component concentrations before clinical recurrence; both had concurrent raised CEA values. By contrast, secretory component was raised in $83 \%$ of those cases considered inoperable.

Conclusions: Although serum secretory component clearly increases in most patients with liver metastases, its clinical value seems questionable because secretory component apparently indicates mainly inoperable hepatic metastases.
\end{abstract}

Rikshospitalet, N-0027

Oslo 1, Norway

D Kvale

P Brandtzaeg

Surgical Department

B, The National

Hospital, Oslo

J N Norstein

Institute for Forensic

Medicine, The

National Hospital,

Oslo

G I Meling,

T O Rognum

Central Laboratory, The Norwegian

Radium Hospital, Oslo

O P Børmer

Cancer Registry of

Norway, Oslo

$F$ Langmark

Correspondence to:

D Kvale

Accepted for publication

28 November 1991

Characterisation of patients

*Males + females.

tMedian (range). metastasis removed at the primary operation. secretory component concentrations and the presence and progression of liver metastasis. $^{12}$

Secretory component is a transmembrane receptor for polymeric IgA (pIgA) and pIgM in secretory epithelial cells, including the colonic epithelium. ${ }^{3}$ It mediates translocation of pIgA and $\mathrm{pIgM}$ into exocrine fluids where its extracellular segments, in conjunction with the respective ligands, become part of the secretory immunoglobulins (SIgA and SIgM) or appear as free secretory component. The small amounts of secretory component appearing in normal peripheral blood exist only as part of circulating SIgA and SIgM due to the large molar excess of pIgA and particularly pIgM in serum. ${ }^{4}$

Secretory component is also expressed by malignant secretory epithelial cells, particularly in colonic carcinomas. ${ }^{56}$ Increased release of secretory component from adenocarcinomas to peripheral blood could conceivably raise the amounts of circulating secretory component. We therefore measured SIgA and SIgM in preoperative serum samples from 100 patients with colorectal carcinoma. A follow up study of the same patient group indicated that secretory component increased in four of the five patients who were later found to have liver metastasis; the median lead time before clinical recurrence was 5.5 months. ${ }^{2}$ However, the small number of patients, and the fact that hepatic resection was not performed, did not permit assessment of the clinical value of these measurements. In view of the recent promising experience with resection of liver metastasis from colorectal carcinoma, ${ }^{78}$ this study aimed to relate concentrations of circulating secretory component in serial serum samples to the clinical outcome of patients subjected to such treatment. The clinical value of secretory component as a tumour marker in colorectal carcinoma could thereby be more firmly established, in relation to early diagnosis of liver metastasis, but particularly as to whether increased secretory component concentrations could indicate if hepatic lesions could be radically removed.

\begin{tabular}{llllllll}
\hline$n=(m+f)^{\star}$ & Age (years) $t$ & $\begin{array}{l}\text { Dukes' stage } \\
(A+B+C+D) \ddagger\end{array}$ & $\begin{array}{l}\text { Primary location } \\
\text { (colon +rectum) }\end{array}$ & $\begin{array}{l}\text { Recurrence time } \\
\text { (months) } \dagger^{\star \star}\end{array}$ & $\begin{array}{l}\text { Recurrences } \\
\text { operated } n(\%)\end{array}$ & $\begin{array}{l}\text { Follow up } \\
\text { (months) } \dagger\end{array}$ & $\begin{array}{l}\text { Samples per } \\
\text { patient } t\end{array}$ \\
\hline $23(11+12)$ & $68(30-83)$ & $4+6+11+2$ & $19+4$ & $8(4-26)$ & $17(74 \%)$ & $16(1-33) 5(1-16)$ \\
\hline
\end{tabular}

†Distant organ metastasis or irremovable tumour was assigned stage D according to Turnbull et al. ${ }^{14}$ Patients with stage D had their

$\star \star$ Time interval between primary operation and clinical recurrence. 


\section{Methods}

Serial serum samples were taken from 23 patients with liver metastases as the only clinical manifestation of recurrence from colorectal carcinoma (table). Cases were selected from a larger group of patients currently subjected to clinical follow up studies.

Liver metastases were confirmed by ultrasound scan, computed tomography, or liver biopsy specimen. The results of surgical treatment of recurrences were classified peroperatively as curative (radical) when no residual tumour was present and the resection margins were microscopically free of tumour.

\section{IMMUNOASSAYS FOR CIRCULATING SECRETORY COMPONENT AND CARCINOEMBRYONIC ANTIGEN (CEA)}

Total serum secretory component was estimated by combining the results from enzyme linked immunosorbent assays (ELISAs) for SIgA and SIgM as described in detail elsewhere. ${ }^{9}$ All patient sera were analysed simultaneously with samples from 54 age and sex matched controls. The upper reference limits for SIgA, SIgM, and secretory component were defined as the 97.5 percentile values in the control group. ${ }^{10}$

CEA was measured in a running laboratory routine by an immunoradiometric assay and with controls as described. ${ }^{11}$

\section{CRITERIA FOR ESTABLISHING INCREASE IN} CIRCULATING SECRETORY COMPONENT AND CEA Secretory component and CEA in serial samples were considered to be increased if the

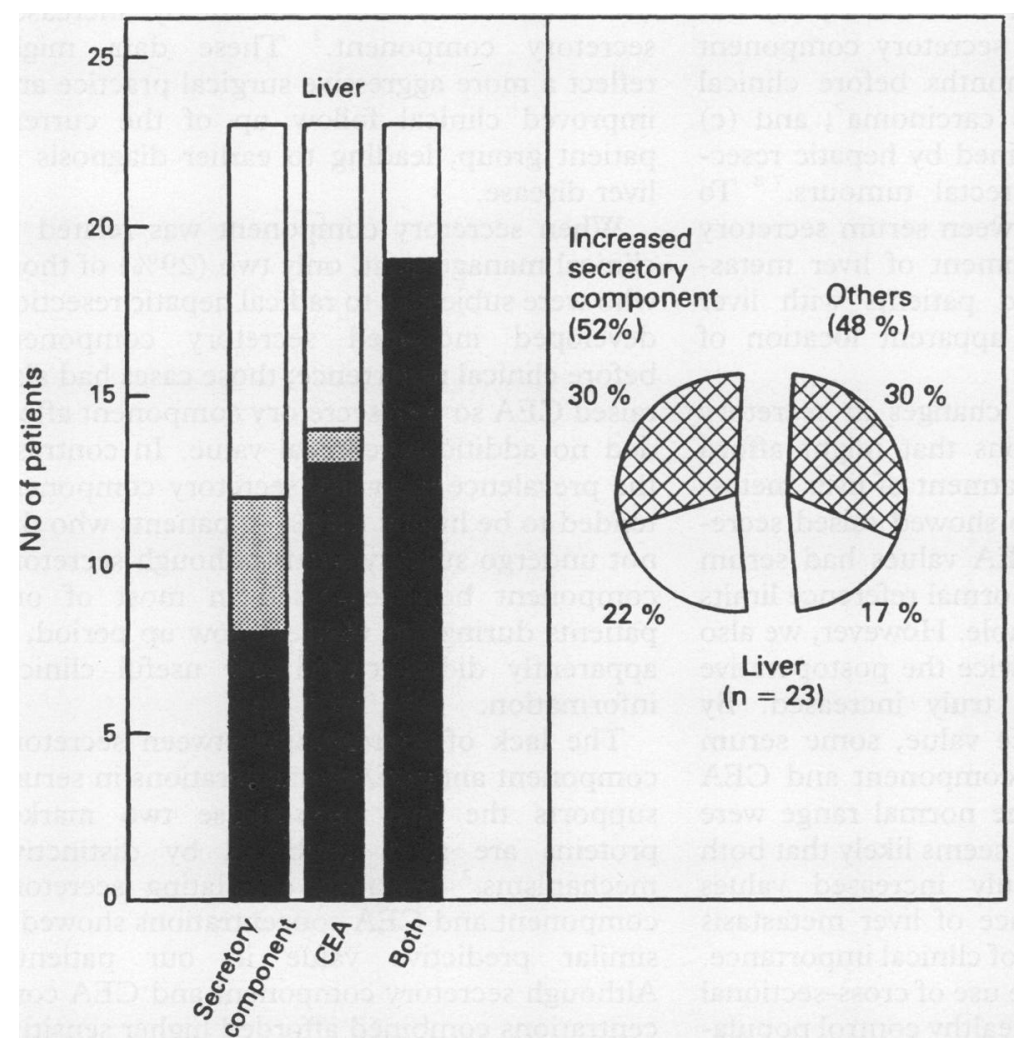

Figure 1 Left panel: Patients with increased serum values of secretory component, or $C E A$, or both before clinical recurrence; first increased serum sample either above upper normal reference values (filled bars), or within reference limits (shaded bars), as indicated. Right panel: Incidence of raised secretory component (unhatched areas) and CEA (hatched areas) in the same patients. serum concentrations were above the upper normal reference limits or permanently increased to at least two-fold the values obtained immediately after the primary operation, even when the raised values were within the normal reference limits.

Lead time was defined as the interval between the first serum sample showing increased secretory component or CEA and the clinical recognition of recurrence.

Comparisons between patient groups were based on the Wilcoxon's two-sample test. Fisher's test of exact probability was used for comparisons of frequency distributions.

\section{Results}

CIRCULATING SECRETORY COMPONENT AND CEA IN RELATION TO RECURRENCE

The upper normal reference limits for SIgA, SIgM, and total secretory component in serum were $42 \mathrm{mg} / 1,33 \mathrm{mg} / \mathrm{l}$, and $11.9 \mathrm{mg} / \mathrm{l}$, respectively, as calculated from the results in the control group.

Altogether, 18 (78\%) patients developed increased secretory component concentrations throughout the whole follow up period. The first positive samples had a median value of $16.3 \mathrm{mg} / \mathrm{l}$ (range $5.4-90.7 \mathrm{mg} / \mathrm{l}$ ). However, only $12(52 \%)$ showed raised secretory component before clinical recurrence with a median initial increased value of $14.6 \mathrm{mg} / 1$ (range $5.4-23.3 \mathrm{mg} / \mathrm{l})$; median lead time was $5 \cdot 2$ months (range 0.1-11.3 months). Four of these 12 patients had samples showing increased secretory component concentrations (according to the criteria defined above) within the normal range (fig 1), but secretory component later continued to increase above the upper normal reference limit in all of them.

CEA was raised before clinical recurrence in $14(61 \%)$ of the patients (fig 1 ) with a median lead time of 2.7 months (range $0.3-14.5$ months). There was no difference in sensitivity or lead times between the secretory component and CEA measurements. However, no correlation was found between an increase in secretory component and CEA. Thus $19(83 \%)$ of the patients developed either increased secretory component CEA values, or both, before recurrence, giving a higher combined sensitivity than that of each maker $(p=0.03)$ and $\mathrm{p}=0.09$, respectively) (fig 1 ).

CIRCULATING SECRETORY COMPONENT AND CEA IN RELATION TO SURGICAL TREATMENT

The patients were divided into three groups: (a) those who were peroperatively classified as radically resected $(n=7) ;(b)$ patients not radically operated on at laparotomy $(n=10)$; and $(c)$ patients who were considered inoperable after clinical examination $(n=6)$.

Only two $(29 \%)$ of the radically resected patients had developed raised circulating secretory component concentrations before recurrence and both had increased CEA at the same time. In contrast, the sensitivity of raised secretory component concentrations tended to be higher in the patients who were not operated on $(83 \%, p=0.08)$. Altogether, $10(63 \%)$ 
Figure 2 Prevalence of increased secretory component and CEA (hatched areas) before clinical recurrence in relation to surgical classification of patients with liver metastasis.
Liver metastasis

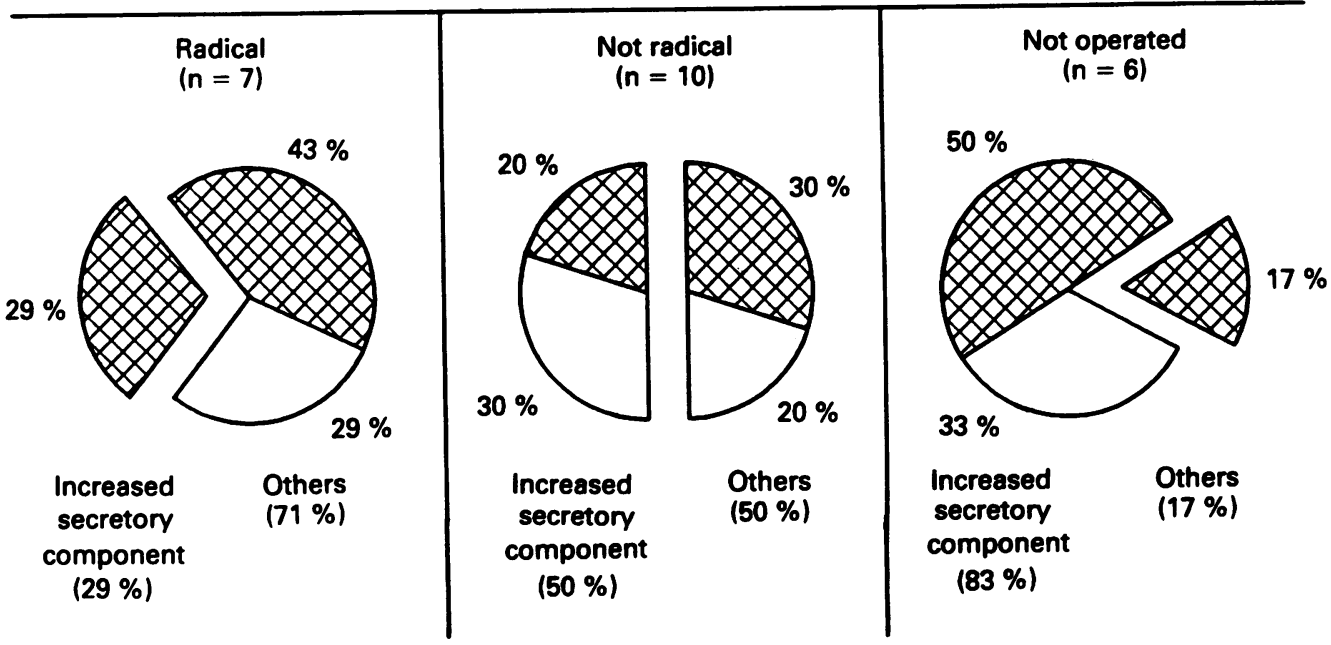

of the patients who were not radically treated had developed increased secretory component (fig 2).

The sensitivity of CEA for patients who underwent radical surgery tended to be higher $(72 \%)$ than for secretory component $(29 \%)$, but not significantly so. Moreover, there was no difference in CEA sensitivity between radically treated cases $(72 \%)$ and the other patients (56\%) (fig 2).

\section{Discussion}

This study was prompted by: (a) a significant association between high secretory component concentrations and liver metastasis ${ }^{1}$; (b) our recent observation that secretory component may increase several months before clinical recurrence of colorectal carcinoma ${ }^{2}$; and (c) the positive results obtained by hepatic resection of secondary colorectal tumours. ${ }^{78}$ To evaluate the relation between serum secretory component and development of liver metastasis per se we studied patients with liver metastasis as the only apparent location of recurrence.

We wanted to detect changes in secretory component concentrations that might afford earlier diagnosis and treatment of liver metastasis. Most patients who showed raised secretory component and CEA values had serum values above the upper normal reference limits in the first increased sample. However, we also defined values at least twice the postoperative concentration as being truly increased. By using this as a reference value, some serum samples with secretory component and CEA concentrations within the normal range were classified as increased. It seems likely that both these definitions of truly increased values might reflect the presence of liver metastasis which, in turn, could be of clinical importance. It may be argued that the use of cross-sectional reference values from a healthy control population is inappropriate for an individual patient over time. ${ }^{12}$ Because our case study material was too small to meet the statistical criteria for estimation of a significant patient specific increase, ${ }^{13}$ we defined an arbitrary two-fold increase of secretory component (and CEA) as a true increase. This limit nevertheless exceeds that of other serum variables. ${ }^{13}$

During the whole follow up period, $78 \%$ of the patients developed increased concentrations of circulating secretory component. This overall result corresponded well with the incidence $(75 \%)$ observed in our previous study. ${ }^{2}$ However, the sensitivity of secretory component for indicating hepatic spread was reduced from $78 \%$ to $52 \%$ before clinical recurrence, with a median lead time of 5.2 months. This result tended to be lower than in our preliminary follow up study, in which four $(80 \%)$ of five recurrences were shown by increased secretory component. ${ }^{2}$ These data might reflect a more aggressive surgical practice and improved clinical follow up of the current patient group, leading to earlier diagnosis of liver disease.

When secretory component was related to clinical management, only two (29\%) of those who were subjected to radical hepatic resection developed increased secretory component before clinical recurrence; those cases had also raised CEA so that secretory component afforded no additional clinical value. In contrast, the prevalence of raised secretory component tended to be higher $(83 \%)$ in patients who did not undergo surgery. Thus although secretory component became raised in most of our patients during the whole follow up period, it apparently did not add any useful clinical information.

The lack of correlation between secretory component and CEA concentrations in serum supports the view that these two marker proteins are shed to blood by distinctive mechanisms. ${ }^{2}$ Notably, circulating secretory component and CEA concentrations showed a similar predictive value in our patients. Although secretory component and CEA concentrations combined afforded higher sensitivity for liver metastasis before clinical detection than either marker alone, this trend did not hold true for the patients who underwent radical resection. 
This study was supported by the Norwegian Cancer Society, Torsted's Fund, Anders Jahre's Fund, The Medical Innovation Foundation, and A/S Freia's Medical Fund. We are grateful for participation of the following hospitals and clinical investigators: Aker Hospital (O C Lunde), Lovisenberg Hospital ( Hognestad), Ullevaal Hospital (E Schlichting), Akershus District General Hospital (E Trondsen), Baerum Hospital (J N Wiig, O P N Grüner), Vestfold Central District Hospital (N Amundsen, A Brudvik, $\mathrm{O}$ Holter), Telemark District Genera Hospital (J B Nilsen), Rjukan Hospital (K Gronneberg), AustAgder District General Hospital (S T Baksaas), Rogaland District General Hospital (A K Olsen), Haugesund County Hospital (I Eikill, K Bakkevold), Nordfiord Hospital (T Hamre Hospital (I Eikill, Bakla Danielsen). We also thank K $\AA$ Eide and A G Myrset for Danielsen). We also thank $K$
assistance with data recording.

1 Kvale D, Rognum TO, Brandtzaeg P. Elevated levels of secretory immunoglobulins $A$ and $M$ in serum of patients with large bowel carcinoma indicate liver metastasis Cancer 1987;59:203-7.

2 Kvale D, Rognum TO, Brandtzaeg P. Early detection of liver metastasis in patients with colorectal carcinoma by increased levels of circulating IgA- and IgM-associated

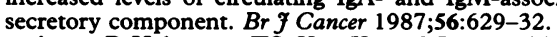

3 Brandtzaeg $\mathrm{P}$, Halstensen TS, Kett K, et al. Immunobiology and immunopathology of human gut mucosa: humoral immunity and intraepithelial lymphocytes. Gastroenterology 1989;97:1562-84.

4 Kvale D, Brandtzaeg P. Does secretory component (SC) exist as free SC in human serum? $f$ Immunol Methods 1988;113:279-81.

5 Rognum TO, Elgjo K, Brandtzaeg P, Ørjasæther H, Bergan
A. Plasma carcinoembryonic antigen concentrations and immunohistochemical patterns of epithelial marker antigens in patients with large bowel carcinoma. $\mathcal{F}$ Clin Patho 1982;35:922-33.

6 Brooks JJ, Ernst CS. Immunoreactive secretory componen of IgA in human tissues and tumors. Am $\mathcal{f}$ Clin Pathol 1984;82:660-5.

7 Hughes KS, Rosenstein RB, Songhorabodi S, et al. Resection of the liver for colorectal carcinoma metastasis. A multi-institutional study of long-term survivors. Dis Colon Rectum 1988;31:1-4.

8 Scheele J, Stangl R, Altendorf-Hofmann A. Hepatic metastasis from colorectal carcinoma: impact of surgical resectasis from colorectal carcinoma: impact of surgical resec-
tion on the natural history. Br $f$ Surg 1990;77:1241-6.

9 Kvale D, Brandtzaeg P. An enzyme-linked immunosorbent assay for differential quantitation of secretory immunoassay for differential quantitation of secretory immuno-
globulins of the $A$ and $M$ isotypes in human serum. $f$ globulins of the $A$ and $M$ isotypes
Immunol Methods 1986;86:107-14.

10 Solberg HE. The theory of reference values, part 5. $f$ Clin Chem Clin Biochem 1983;21:749-60.

11 Børmer OP, Nustad K. Selection of monoclonal antibodies for use in an immunometric assay for carcinoembryonic antigen. F Immunol Methods 1990;127:171-8.

12 Harris EK, Yasaka T, Horton MR, Shakarji G. Comparing multivariate and univariate subject-specific reference regions for blood constituents in healthy persons. Clin Chem 1982;28:422-6.

13 Harris EK, Yasaka T. On the calculation of a "Reference Change" for comparing two consecutive measurements. Clin Chem 1983;29:25-30.

14 Turnbull RB, Kyle K, Watson FR, Spratt J. Cancer of the colon: The influence of no-touch isolation technique on survival rates. Ann Surg 1967;166:420-5. 